\title{
Cancer detection by fruit fly olfaction
}

Cancer cells and non-cancer cells differ in metabolism and emit distinct volatile compounds, allowing them to be distinguished by their scent. Insect odorant receptors are excellent chemosensors with high sensitivity and a broad receptive range. Putting these two ideas together, collaborating scientists from University of Konstanz (Germany) and University of Rome (Italy) investigated the potential of using the fruit fly's olfactory system to detect cancer cells. In previous work, dogs have been used for cancer chemosensing, but although their olfaction may be sensitive and accurate, the approach has the limitation that its 'readout' is only indirect, based on the animal's behavior in combination with a human trainer and interpreter. This limitation means that results of chemosensing experiments using dogs are difficult to quantify and therefore can't be reliably used in clinical diagnostics. In contrast, the new fruit fly system avoids a behavioral readout and instead uses a direct readout of the insect's antenna responses obtained by calcium imaging. "What really is new and spectacular about this result is the combination of objective, specific and quantifiable laboratory results and the extremely high sensitivity of a living being that cannot be matched by electronic noses or gas chromatography," explained Giovanni Galizia, leader of the project, in a press release.

In fruit fly antennae, single odorant molecules dock to receptor neurons, activating them. The scientists used calcium imaging to record the pattern of neuron activation in response to scent samples from cultures of cancer cells, cultures of healthy cells or culture medium. The response patterns were different for cancerous versus healthy cells and were conserved among individual flies. Therefore, the response patterns could be used to distinguish samples of healthy cells from those of cancerous cells and even to distinguish two different groups of cancer samples according to their proliferation rates (Sci. Rep. 4, 3576; 2014).

The results serve as proof of the concept that the fruit fly olfactory system can detect

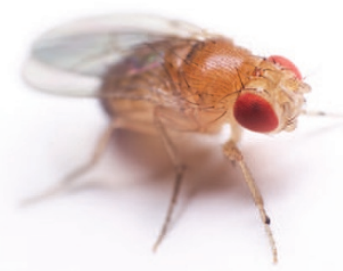

and discriminate among medically relevant odors at low concentrations and is therefore suitable for medical applications. "The high sensitivity of the natural olfactory receptors, paired with the quickness with which we can generate these test results, might lead to the development of a cheap, fast and highly efficient pre-screening that can detect cancer cells well before we can discover them with the present diagnostic imaging techniques," noted Galizia. In the future, the scientists plan to work on integrating the odorant receptors into artificial systems with the potential for real-time readouts.

Monica Harrington

\section{PARKINSON'S DRUG COULD TREAT DIABETIC RETINOPATHY}

Diabetes can result in diabetic retinopathy, causing abnormal color vision and decreased contrast sensitivity, effects that have been consistently replicated in diabetic rodent models. Doctors had previously thought the impairment of vision came from damage to the blood vessels induced by high blood sugar, but it is increasingly recognized that retinal dysfunction predates such vascular lesions.

Retinal dopamine directly modulates multiple aspects of vision through activation of selective receptors and retinal pathways. Disruption in the retinal dopaminergic system has been observed in diabetes, though its potential contribution to the pathogenesis of diabetic retinopathy remains unclear. New research elucidates this contribution and suggests that a dopamine-restoring drug already used to treat Parkinson's disease, called L-DOPA, may also be used to treat diabetic retinopathy.

Researchers led by Machelle Pardue (Atlanta VA Medical Center, GA) and Michael Iovone (Emory University, Atlanta, GA) studied rats and mice made diabetic by treating them with streptozotocin (STZ), a substance that is toxic to insulin-producing cells in the pancreas. The induced diabetes significantly reduced retinal dopamine levels by 4 weeks or 5 weeks after STZ injections in rats and mice, respectively. Next, they tested the vision of diabetic mice by placing them on a platform surrounded by four computer screens that displayed rotating black and white lines. Spatial acuity was assessed by gradually making the lines finer, and contrast sensitivity was determined by reducing the contrast between the black and white lines, both until the mice stopped tracking the rotating lines. Diabetic mice showed both reduced spatial frequency thresholds and reduced contrast sensitivities.

In another experiment, mice with dopamine selectively deleted in the retina also showed reduced spatial acuity and contrast sensitivity, confirming the key role of dopamine in vision. When these mice were made diabetic with STZ, their vision did not deteriorate further, confirming that the visual dysfunction seen in diabetic mice is linked to a loss of dopamine.

In contrast, treating the diabetic mice with L-DOPA delayed and mitigated the visual deficits; spatial frequency thresholds only diminished after 4 weeks, and contrast sensitivities only after 6 weeks (J. Neurosci. 34, 726-736; 2014). L-DOPA treatment also rescued the visual impairments of the mice genetically lacking retinal dopamine by restoring levels to those of control mice.

Said Pardue of the findings, "There was some evidence already that dopamine levels were reduced in diabetic retinopathy, but what's new here is we can restore dopamine levels and improve visual function in an animal model of diabetes."

Kara Rosania 